\title{
Industry Partnerships: Disease Management Programs Flourish
}
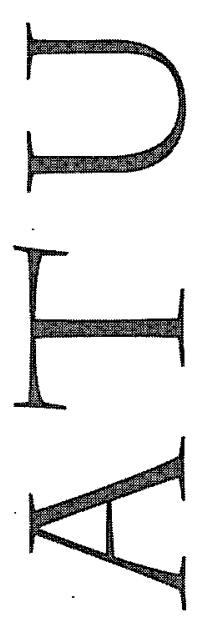

Diverse organizations are becoming partners in programs to manage specific, costly diseases and conditions. Drug manufacturers, pharmacy benefit managers, and managed care organizations are launching disease management
programs that involve active participation from pharmacists in many settings. agers, and managed care organizations are launching disease management
programs that involve active participation from pharmacists in many settings.
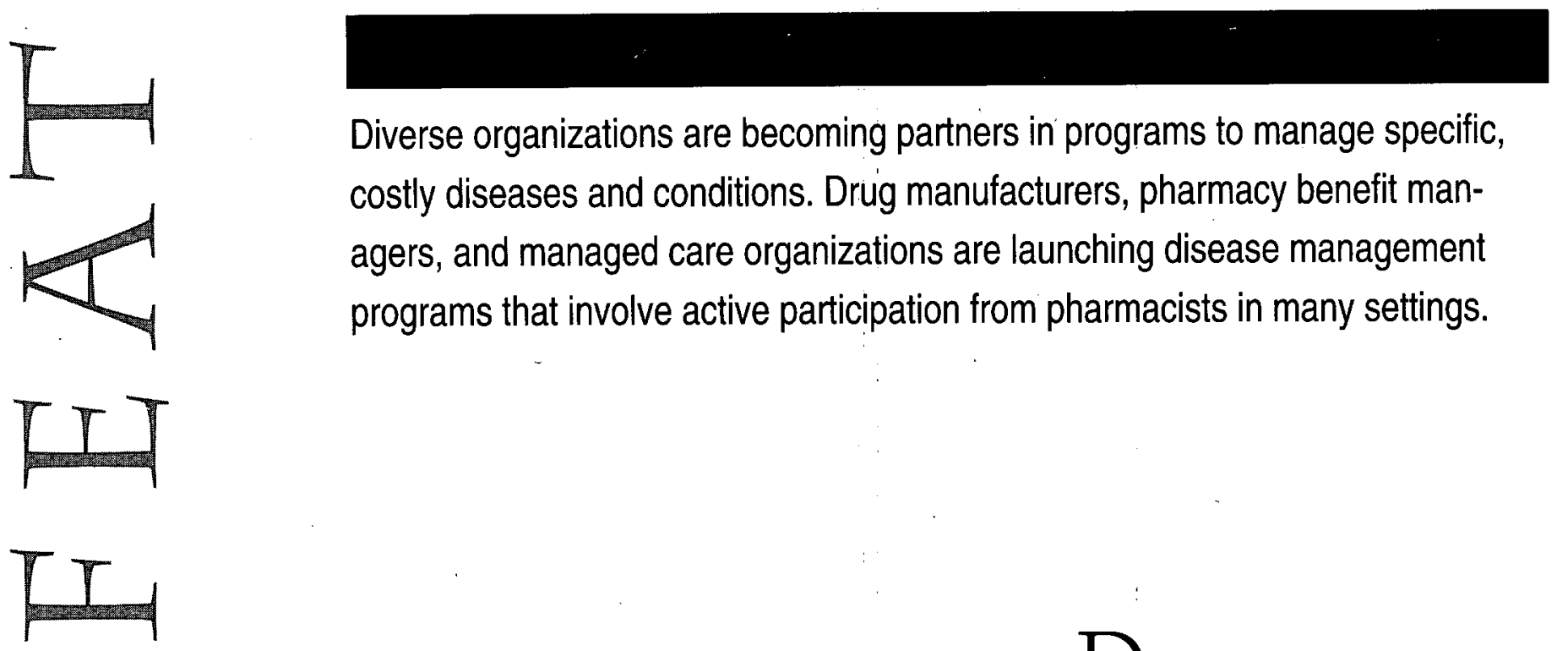

\section{MARY JANE GORE}

$\mathrm{D}$ isease management (DM) is not just a buzz phrase for the times. Such programs are being pursued and implemented by a wide variety of organizations nationwide, driven by the push to lower the cost of American medicine.

These programs strive to control the costs of several specific diseasesmost often, asthma, diabetes, hypertension, hyperlipidemia, and cardiac disease. The diseases being managed or prevented by such programs are generally very prevalent and/or very expensive to treat.

This article will examine how some companies have chosen to tackle disease management, who their partners/customers are, how the programs are operated and funded, and how they measure program success.

Continued on page 166 


\section{INDUSTRY PARTNERSHIPS ABOUND}

Pharmacists in a variety of settings are finding themselves part of DM programs with shared responsibility for administration and other aspects of the program:

$\nabla$ Pharmaceutical manufacturers are partnering with employers, pharmacy . benefit management firms (PBMs), managed care organizations (MCOs), and other clients;

$\nabla$ PBMs are launching multidisciplinary programs with MCOs;

$\checkmark$ MCOs, particularly staff-model HMOs, have traditionally performed disease management for their members; $\nabla$ Home care companies are finding industry partners (e.g., Caremark International; Apria Healthcare); and

$\checkmark$ Pharmacy professionals themselves are developing DM programs (e.g., Health Today, run by several consultant pharmacists and based in Boston).

According to Business \& Health, "the most vocal advocates of disease management are the pharmaceutical managers and the pharmacy benefits managers." Several pharmaceutical firms, including Glaxo Wellcome, Pharmacia \& Upjohn, Zeneca, Eli Lilly, Schering, and Wyeth-Ayerst, have recently entered into partnerships with others to provide disease management services.

PBMs are also becoming involved in DM programs, most of which are relatively new or under development. PCS Health Systems, a wholly owned subsidiary of Eli Lilly, has assumed responsibility for Lilly's Integrated Disease Management (IDM) Group's work. Diversified Pharmaceutical Services in Minneapolis works with $\mathrm{HMO}$ clients to manage a diabetes program that involves a multidisciplinary approach to improvement, including certified diabetes educators. Medco Containment Service has an optimal outcomes program that distributes educational materials to diabetes sufferers. Recently, Merck-Medco and Wyeth-Ayerst teamed up in a joint venture to develop DM programs in several therapeutic areas, including prenatal care, menopause, os- teoporosis, family planning, arthritis, and cardiac arrhythmias. Other programs, no doubt, are under development across the country.

"The rationale for disease management and prevention is so compelling that we have not identified anyone who does not have an interest in it," says Wilbur Pittinger, Senior Vice President of Health Management Services for PCS Health Systems in Scottsdale, Ariz.

\section{HOW DM PROGRAMS EVOLVE -}

Bill Baicy, Vice President and General Manager of Glaxo Wellcome's new Care Management Division in Research Triangle Park, North Carolina, shared many insights into the development and monitoring of his company's existing and future programs. Glaxo Wellcome is using clinical experts in several different therapeutic areas and input from customers to offer patients a variety of programs. Some efforts are directed at asthma, gastrointestinal disease, acid peptic disease, headache management, and oncology support (managing symptoms stemming from chemotherapy, including anemia and profound emesis). Under development are programs for managing HIV and herpes.

Glaxo Wellcome "always tries to make its programs a joint effort with customers, because it is much more effective if we have people at the company who have a vested interest in seeing that the programs are successful," Baicy says. However, the manufacturer may take the lead in developing and disseminating educational materials or performing a review of the patient in his home environment. Glaxo has among its 50-person DM staff "customer accounts" representatives who review the customers' needs and suggest various programs to meet customer needs. One group examines customer data to analyze where they are spending money and measures this information through a benchmarking program with data from other companies. Glaxo also has medical professionals on staff who help to implement programs at individual customer sites.

Two years ago, Eli Lilly developed its IDM Group, and the first products were rolled out in 1994. The IDM work was later transferred for operational reasons to PCS, the corporate center for activities of disease prevention and management. "We're coordinating efforts to maximize the synergies of all of the efforts underway [at Lilly and PCSI," Pittinger says. "There's a lot going on globally within the corporation in the U.K., Germany, and New Zealand," countries that are seeking the same kind of capabilities. To date, PCS is working on DM programs in diabetes, asthma, depression, infectious diseases, hyperlipidemia, gastroesophageal reflux disease, and peptic ulcer disease.

The IDM Group developed by Lilly is just one component of PCS' Health Management Division, Pittinger says. An internal development group generates clinical interventions and guidelines where necessary. In addition, there is a medical implementation group, an outcomes research department, clinical information system division (works on information technology interfaces), clinical operations group of pharmacists, and a clinical services group of physicians.

Pharmacia \& Upjohn has a wholly owned subsidiary, Greenstone Healthcare Solutions (also based in Kalamazoo, Michigan), that takes a different approach to DM. Instead of offering set programs or products, Greenstone "enables" disease management providers to develop and improve their programs, says Sheri Staak, Director of Health Management for Greenstone. "When you are tracked horizontally and treated as an individual across an entire system, you are better able to be treated appropriately with the appropriate outcomes," she stresses. The Greenstone approach to DM has a strong foundation in information systems that helps to identify the specific problem and the solution, be it a barrier in the system or a patient compliance problem. Again, diabetes is the major disease concern, followed by hyperlipidemia, chronic

Continued on page 170 

sion occurred in $1.4 \%$ of patients treated with fosinopril monotherapy. Hypotension or orthostatic hypotension was a cause for discontinuation of therapy in $0.1 \%$ of patients. Dermatologic: Urticaria, rash, photosensitivity, pruritis. Endocrine/Metabolic: Gout, decreased libido. Gastrointestinal: Pancreatitis, hepatitis, dysphagia, abdominal distention, abdominal pain, flatulence, constipation, heartburn, appetite/weight change, dny mouth. Hematologic: Lymphadenopathy. Immunologic Angioedema. (See WARNINGS: Angioedema). Musculoskeletat. Arthralgia, musculoskeletal pain, myalgia/muscle cramp. Nenous/Psychiatric. Memory disturbance, tremor, confusion, mood change, paresthesia, sleep disturbance, drowsiness, vertigo. Respiratory, Bronchospasm, pharyngitis, sinusitis/rhinitis, laryngitis/hoarseness, epistaxis. A symptom-complex of cough, bronchospasm, and eosinophilia has been githoar in two patients treated with fosinopril. Special Senses. Tinnitus, vision disturbance taste disturence controlled clinical trials (361 MONOPRIL-treated patients) the usual duration of therapy was 3-6 controlled cinical trials (J61 MONOPRIL-treated patients), the usual duration of therapy was 3-6 months. Discontinuation due to any clinical or laboratory adverse event, except for heart failure, were $8.0 \%$ and $7.5 \%$ in MONOPRIL-treated and placebo-treated patients, respectively. The most frequent reason for disconthuation of MONOPRIL was angina pectoris (1.1\%). Significant hypotension after the first dose of MONOPRL first dose hypotension. Clinical adverse events probably or possibly related or of uncertain relationship to therapy, occurring in at least $1 \%$ of patients treated with MONOPRIL and at least as common as the
placebo group, in placebo-controlled trials are shown in the following table on page xx.

Clinical Adverse Events in Placebo-Controlled Trlais (Heart Failure)

$\begin{array}{lcc} & \begin{array}{c}\text { MONOPRIL } \\ \text { (N=361) } \\ \text { Incidence } \\ \text { (Discontinuation) }\end{array} & \begin{array}{c}\text { Placebo } \\ \text { (N=373) } \\ \text { Incidence } \\ \text { (Discontinuation) }\end{array} \\ \text { Dizziness } & 11.9(0.6) & 5.4(0.3) \\ \text { Cough } & 9.7(0.8) & 5.1(0.0) \\ \text { Hypotension } & 4.4(0.8) & 0.8(0.0) \\ \text { Musculoskeletal Pain } & 3.3(0.0) & 2.7(0.0) \\ \text { NauseaNomiting } & 2.2(0.6) & 1.6(0.3) \\ \text { Diarrhea } & 2.2(0.0) & 1.3(0.0) \\ \text { Chest Pain (non-cardiac) } & 2.2(0.0) & 1.6(0.0) \\ \text { Upper Respiratory Infection } & 2.2(0.0) & 1.3(0.0) \\ \text { Orthostatic Hypotension } & 1.9(0.0) & 0.8(0.0) \\ \text { Subjective Cardiac } & 1.4(0.6) & 0.8(0.3) \\ \text { Rhythm Disturbance } & 1.4(0.3) & 0.5(0.0) \\ \text { Weakness } & & \end{array}$

obstructive pulmonary diséase, and congestive heart failure.

Greenstone often cleans claims data for inputting and other errors and "mines" the claims data, which "has a lot of answers in it," Staak says. The various information tools that Greenstone uses have the capability to tell the provider or payer-both of whom are customers-what the problem is, based on an extensive review of data.

The Greenstone program has grown to be marketed by a sales force of 20 people, five of whom focus on the payer side, the rest on the provider side. While the sales force is the primary means for marketing products, Greenstone has also established a World Wide Web site on the Internet

(http: Nwww.sapien.netigreenstonel) that includes a forum for "general chat sessions about disease management," Staak says.

The following events also occurred at a rate of $1 \%$ or more on MONOPRIL (Fosinopril Sodium) but occurred on placebo more often: fatigue, dyspnea, headache, rash, abdominal pain, muscle cramp, angina pectoris, edema, and insomnia. The incidence of adverse events in the elderly ( $\geq 65$ years old) was similar to that seen in younger patients. Other clinical events probably or possibly related, or of uncertain relationship to therapy occurring in 0.4 to $1.0 \%$ of patients (except as noted) treated with MONOPRIL in controlled clinical trials $(N=516)$ and less frequent, clinically significant events include (listed by body system): Generat: Fever influenza weight gain, hyperhidrosis, sensation of cold, fail, pain. by body system): General: Fever, influenza, weight gain, hyperhidrosis, sensation of cold, fail, pain. Cardiovascular: Sudden death, cardiorespiratory arrest, shock (0.2\%), atrial rhythm disturbance, cardiac rhythm disturbances, non anginal chest pain, edema lower extremity, hypertension, syncope, conduction disorder, bradycardia, tachycardia. Dermatologic: Pruritus. Endocrine/Metabolic: Gout, sexual dysfunction. Gastrointestinal: Hepatomegaly, abdominal distension, decreased appetite, dry mouth, constipation, flatulence. Immunologic. Angioedema. (0.2\%). Musculoske/etat. Muscle ache, swelling of an extremity, weakness of an extremity. Nervous/Psychiatric. Cerebral infarction, TIA, depression, numbness, paresthesia, vertigo, behavior change, tremor. Respiratory. Abnormal vocalization, rhinitis, sinus abnormality, tracheobronchitis, abnormal breathing, pleuritic chest pain. Special Senses. Visual disturbance, taste disturbance. Urogenital: Abnormal urination, kidney pain.

Fetal/Neonatal Morbidity and Mortality See WARNINGS: Fetal/Neonatal Morbidity and Mortality. Potential Adverse Effects Reported with ACE Inhibitors Body as a whole: Anaphylactoid reactions (see WARNINGS: Anaphylactoid and possible related reactions and PRECAUTIONS: Hemodialysis). Othe medically important adverse effects reported with ACE inhibitors include: Cardiac arrest; eosinophillic pneumonitis; neutropenia/agranujocytosis, pancytopenia, anemia (including hemolytic and aplastic) thrombocytopenia; acute renal failure; hepatic failure, jaundice (hepatocellular or cholestatic); symptomatic hyponatremia; bultous pemphigus, exfoliative dermatitis; a syndrome which may include: arthralmatic hyponatremia; bullous pemphigus, exiar rash or other dermatologic manifestations, a positive ANA, leukocytosis, eosinophilia, or an elevated ESR. Laboratory Test Abnormalities Serum Electrolytes: ANA, leukocytosis, eosinophilia, or an elevated ESR. Laboratory Test Abnormalities Serim, with diuretHyperkalemia, (see PRECAUTIONS); hyponatremia, (see PRECAUTIONS: Drug Interactions, With diuretiCS). BUN/Serum Creatinine: Elevations, usually transient and minor, of BUN or serum creatinine have been observed. In placebo-controlled clinical trials, there were no signiticant differences in the number of patients experiencing increases in serum creatinine (outside the normal range or 1.33 times the pretreatment value) between the fosinopril and placebo treatment groups. Rapid reduction of longstanding or markedly elevated blood pressure by any antihypertensive therapy can result in decreases in th glomerular filtration rate and, in turn, lead to increases in BUN or serum creatinine. (See PRECAUTIONS: General.) Hematology: In controlled trials, a mean hemoglobin decrease of $0.1 \mathrm{~g} / \mathrm{dL}$ was observed in fosinopril-treated patients. In individual patients decreases in hemoglobin or hematocrit were usually transient, small, and not associated with symptoms. No patient was discontinued from therapy due to the development of anemia. Other: Neutropenia (see WARNINGS), leukopenia and eosinophilia. Liver Function Tests: Elevations of transaminases, LDH, alkaline phosphatase and serum bilirubin have bee reported. Fosinopril therapy was discontinued because of serum transaminase elevations in $0.7 \%$ of patients. In the majority of cases, the abnormalities were either present at baseline or were associated with other etiologic factors. In those cases which were possibly related to fosinopril therapy, the elevations were generally mild and transient and resolved after discontinuation of therapy.

OVERDOSAGE

Oral doses of fosinopril at $2600 \mathrm{mg} / \mathrm{kg}$ in rats were associated with significant lethality. Human overdoses of tosinopril have not been reported, but the most common manifestations of human tosinopril ovedosa is likely to be hypotension. Laboratory determinations of serum levels of fosinoprilat and its metabolites are not widely available, and such determinations have in any event, no established role in the management of fosinopril overdose. the management of tosinopril overdose. No data ane al a fosinopril and its metabolites. Fosinoprilat is poorly removed from the body by both hemodialysis and peritoneal dialysis. Angiotensin ll could presumably serve as a specific antagonist-antidote in the setting of fosinopril overdose but angiotensin il is essentially.unavailable outside of scattered research facilities. Because the hypotensive effect of fosinopril is achieved through vasodilation and effective hypovolemia, it is reasonable to treat fosinopril overdose by infusion of normal saline solution. Consult package insert before prescribing Monopril (fosinopril sodium).

F4-B001A-4-95 Revised: April 1995

\section{WHO BUYS DISEASE MANAGEMENT?}

Pittinger says that typically PCS has developed customer relationships with payers, generally with MCOs, especially HMOs, and also with integrated delivery systems (provider organizations). He says that PCS Health Systems has access to a large employer base of customers, and the PCS Health Management Services Division is now trying "to demonstrate clinical intervention capability to them in order to improve the quality and cost-effectiveness of care for employees." Pittinger says that more often, PCS is approached by MCOs and information technology firms that seek clinical capability for applications.

Typically, PCS works with customers on a risk-sharing basis. "We need their help to implement the programs, and they need our help to develop the programs," Pittinger says. "That is not to say that we have not done programs on a feefor-service basis, however."

Baicy says it is difficult to categorize its partners in disease management. These range from large employers (who obtain services for employees directly at the work site through a managed care provider or PBM), managed care organizations (IPAs, HMOs, and staff-model HMOs), and PBMs. Many of its programs are more hands-on, administered through telephone contact, he says.

To date, Glaxo Wellcome is offering its DM programs through two channels: for sale, through a contract to provide certain programs for patients at a set fee; and second, through a "shared benefit," Baicy says. In the first case, claims or other data might identify, say, 300 patients with asthma. Glaxo will offer a program for those 300 patients that would cost a flat fee.

In the second case, Glaxo helps to design a more comprehensive, specific program for the 300 patients by establishing a baseline for costs of care, at say $\$ 300,000$ over one year-for hospitalization, drugs, and lost productivity and other indirect costs. Glaxo designs a program to improve care to the patients and to lower costs. "We would share the benefit of the lowered cost," he explains.

'This partnership would involve both Glaxo and the cus-

Pharmaceutical Research Institute.

Bristol-Myers Squibb Company 
tomer working closely together for Glaxo to be able to implement the program, gain the support of providers who should monitor these patients more aggressively, and put the patients through educational programs, Baicy says. More severe patient cases might require an ancillary health care professional such as a respiratory therapist or a nurse practitioner.

Greenstone's customers most often are Fortune 100 employers and insurance companies, although Greenstone is now targeting Fortune 500 companies. "We have had interactions with Fortune 100 insurance companies on the payer and provider sides, and health care consultants and benefits firms (such as Hewitt). We have also worked with managed health care plans, such as HMOs, group practices, and hospital systems," Staak says.

Greenstone handles anywhere from $10 \%$ to $99 \%$ of the program logistics and operations, depending on the customer's desire for involvement. "These customers are short on people to run these kinds of programs, full-time equivalents, because they operate on thin margins," Staak notes. "They don't want to have to hire people to do it."

If an HMO has its own DM program under development, it may elect to improve its program with advice and support from a manufacturer, Pittinger says. With DM and cost savings on everyone's mind, various types of partnerships and programs are springing up today

\section{DM PROGRAM GOALS}

All of the companies interviewed for this article mentioned two-part goals for their DM initiatives: first, to improve patient health and care, and second, to reduce costs of care.

Baicy of Glaxo Wellcome says that "there are [disease management] programs that are just focused on a drug or on care providers." He says that such programs must focus instead on patients who need to gain knowledge about disease progression, and how it affects the patients and their families. "They need the knowledge so they can take on more responsibility," Baicy told JMCP.

Some have suggested that an unspoken goal of disease management programs is to shift market share for medications. Staak is of the opinion that "other manufacturers who have started disease management businesses are creating a marketing tool in which to wrap value-added services around a solution, and their solution is the medication." While Greenstone says this is "not a bad strategy," she sells services for revenue, and the services are not wrapped around a solution that is a drug, she says.

Baicy says that Glaxo has not "done in-depth analysis as far as share shift because it is not a focus of my group, nor is it a goal." However, he readily notes that Glaxo has centered its programs in areas of the company's drug and clinical expertise, because pharmaceutical customers have requested increasing assistance with aspects of various chronic diseases.

Pittinger says the PCS objective is "to be certain that the customer, whether it's in the open formulary situation or the preferred drug formulary situation, is receiving the best pharmaceutical for the condition of the patient being treated."

He notes that this coming year, PCS plans to roll out educational programs for people with diabetes and asthma that do not revolve around a medication. Behavior modification is the major thrust of these educational programs.

\section{EXISTING PROTOCOLS USED, NEW PROTOCOLS CREATED}

Baicy says that for the diseases targeted by Glaxo, existing protocols are available. In the case of asthma, many organizations have adopted the NIH guidelines for treatment. "We basically work with those guidelines," he says.

At Greenstone, three types of clinical protocolș are used: evidence-based; consensus-based; and research-based. For the lattermost category of protocols, Greenstone works with the customer to build the protocols inside the cus- tomer's system to "gain buy-in of physicians and the knowledge of physicians, and to continuously improve care," Staak says. She says Greenstone has purchased or used thousands of evidence-based protocols (developed through a search of medical literature), as well as protocols devised through a medical consensus process, such as that of the American Psychiatric Association and other organizations. The customized protocols devised by Greenstone for clients are not proprietary, she says.

\section{ROADBLOCKS TO IMPLEMENTATION}

Probably the most common roadblocks to implementation of DM programs are physician incentive and reward systems, which are not aligned properly for motivation to get to outcomes management, Staak says. Often, processes within a business are also not aligned to get to the best outcomes on behalf of a patient. Implementation can be difficult because it not only depends upon the health care system alignment, but also on the politics of corporate life, economics, and other factors in a very dynamic marketplace.

Baicy says that, as with any new endeavor, the road is not always smooth, but this area is hard to argue against. "It really evolved out of working with customers through our pharmaceutical business," he says. "Many of them were asking for more help in managing certain diseases, such as asthma. We evolved different levels of service to meet customer needs."

Baicy says the big roadblocks have been organizational-getting the right people in place, finding the right people, defining the jobs that need to be accomplished. Because Glaxo has to be able to respond to needs nationwidebecause it is a company that serves "just about every health care provider in the U.S." The problem is how to meet with all of those customers on a timely basis. 


\section{MEASURES OF PROGRAM SUCCESS}

At the heart of all of these DM programs is a means for measuring program success, usually by a variety of outcomes measures. Staak says that Greenstone outcome measures vary naturally according to the disease. For diabetes, a primary outcome would be blood glucose control, which in turn reduces complications and the economic impact on the system. The company works with the National Commission on Quality Assurance (NCQA) and the Joint Commision for Accreditation of Hospital Organizations (JCAHO) for the purposes of standardizing its measurements.

Baicy says that customers will tell Glaxo, "fairly readily," whether it has done its DM job or not. "Almost every program has a tracking mechanism that will review if patients have changed their knowledge level and educational level, changed behavior, or changed hospitalization usage rates. There are soft measures and hard measures put in place."

Pittinger stressed the central role of outcome measures in DM, and the critical role of information technology in providing data for these programs.
Again, outcomes depend on the disease and the program. Some outcomes measured include:

$\nabla$ Measuring and enhancing diagnosis capability;

$\nabla$ Implementing the most effective treatment plan for a given diagnosis and set of conditions;

$\checkmark$ Measuring patient compliance with the treatment plan; and

$\nabla$ Measuring functional status of the patient. Has their quality of life improved as well as the clinical condition?

"All of those things are important, and we use continuous quality improvement approaches," Pittinger says. "We are always looking at outcomes to continuously improve what we have implemented and measure a difference."

\section{PHARMACIST'S ROLE IN DM}

Pharmacists should be involved in providing care in a comprehensive disease management program, Baicy says. For example, community pharmacists in Glaxo's asthma program are instrumental in teaching proper inhaler use. In other DM programs, specialized pharmacists participate in the oncology support program wherein patients are treated at a cancer center. There, an oncology pharmacist plays a role that is not open to community pharmacists. In all of the DM programs, however, pharmacists should be active, involved participants, Baicy stresses.

Pittinger says pharmacists "absolutely" have a large role in DM programs. "One of the areas that reports to me is the Clinical Operations Area, which is the performance-based clinical intervention group of pharmacists at PCS. They analyze customers' formularies and formulary utilization." These pharmacists examine drug-use factors, as well as prior authorization, and provide clinical intervention information that goes back to customers to improve the quality of care to patients as well as to reduce expenditures associated with care.

Pharmacists can intervene through clinical intelligence built into the information system, or a pharmacist may get directly involved through PCS Health Systems," Pittinger says. "I think the opportunity for clinical intervention will grow, and move upstream, as close to the point of service as possible, moving into the physician's office and to the prescribing physician at the time that a clinical activity occurs."

\title{
$\checkmark$ References \\ 1. Mandelker J. Managing chronic disease. Bus Health 1994; 12(11):45-50.
}

\section{The AMCP}

\section{Medicare/Medicaid Pharmacy Conference}

\author{
The Practical Advice \\ You Need to Keep Up With \\ Emerging Rules and Trends
}

\author{
January 28-30, 1996 \\ The Stouffer Renaissance Harborplace Hotel \\ Baltimore, MD
}

Space is limited. Register now. For information or a brochure, call 1-800-TAP-AMCP. 